\title{
Role of primary and secondary prevention in atopic dermatitis
}

\author{
Magdalena Oszukowska, Iwonna Michalak, Katarzyna Gutfreund, Wojciech Bienias, Marta Matych, Anna Szewczyk, \\ Andrzej Kaszuba
}

Department of Dermatology, Paediatric Dermatology and Oncology, Medical University of Lodz, Lodz, Poland Head of the Department: Prof. Andrzej Kaszuba MD, PhD

Postep Derm Alergol 2015; XXXII (6): 409-420

DOI: 10.5114/pdia.2014.44017

\begin{abstract}
Atopic dermatitis (AD) is a serious epidemiological problem in industrialized countries. The incidence of AD has increased considerably over the last 30 years. Atopic dermatitis is a chronic, recurrent, inflammatory skin disease accompanied by strong itching. It is characterized by typical features depending on age. The parents of children suffering from AD must be prepared to change their lifestyle. They should avoid factors which can promote skin lesions and apply appropriate, regular skin care. The article describes primary prevention of AD as well as prophylactic measures to avoid skin eczema. It presents the role of infections, vaccinations, breastfeeding and the influence of domestic animals, house renovation and moulds on development of AD. The article also describes the significance of the epidermal barrier, skin colonization by microbial agents, pruritus, stress, food and inhalant allergy among people who suffer from $A D$.
\end{abstract}

Key words: atopic dermatitis, primary prevention, secondary prevention.

\section{Introduction}

Atopic dermatitis (AD, endogenous eczema) is a chronic, recurrent and inflammatory skin disease, often running in a family, which is accompanied by severe itching and typical skin lesions depending on age. In infants, the first eczematous lesions occur on the cheeks. In early childhood, the lesions occupy the nape of the neck, dorsal surfaces of the limbs and the joint flexural areas. In later years, apart from active eczematous lesions, lichenified plaques may dominate in the area of joint flexion, head and neck. There is a strong association between $A D$ and allergic respiratory diseases, asthma, allergic rhinitis and conjunctivitis.

Atopic dermatitis poses an important epidemiological problem in industrialized countries. The AD prevalence is different depending on the age and affects $15-30 \%$ of children and $2-10 \%$ of adults. Over the past three decades in industrialized countries, there has been a two- or even three-fold increase in the incidence of AD. In total, $45 \%$ of $A D$ cases are recorded in the first 6 months of life, $60 \%$ in the first year of life and $85 \%$ before 5 years of age [1].

Atopic dermatitis is characterized by chronic inflammation, impairment of the cutaneous-epidermal barrier and hypersensitivity to food and environmental allergens induced by immunoglobulin E (IgE). More than $50 \%$ of children with symptoms of AD during the first 2 years of life do not have any signs of hypersensitivity associated with IgE, but this varies during the course of the disease. Adult-onset AD can be also distinguished, in which IgErelated hypersensitivity does not usually occur [1]. It is currently believed that damage to epidermal cells leading to the epidermal barrier dysfunction is the primary one, and immunological aspects are a secondary phenomenon, which, however, further promote and support the development of AD [1].

The primary risk factor for $A D$ in children is the occurrence of atopic diseases in parents. Approximately 20-30\% of children whose one parent is atopic and about $40-50 \%$ of children with two atopic parents develop atopic allergy. In only $10 \%$ of children suffering from atopic disease no symptoms of atopy have been found in their parents [2, 3]. A stronger risk factor for AD is thought to be associated with the presence of atopy in the mother rather than the father [4]. However, a significant increase in the incidence of $A D$ in developed countries over the past three decades cannot be explained by genetic predisposition only. It seems to be reasonable

Address for correspondence: Magdalena Oszukowska MD, Department of Dermatology, Paediatric Dermatology and Oncology, Medical University of Lodz, 1/5 Biegańskiego St, 91-347 Lodz, Poland, phone: +48 48691222 965, e-mail: wertynka@yahoo.com Received: 21.10.2013, accepted: 3.04.2014. 
that there are other factors that influence the development of AD. This is clearly evidenced by the fact that immigrants become prone to develop $A D$ in the environment to which they have moved [5].

\section{Primary prevention}

Primary prevention applies to children in whom no signs of the disease have been observed yet, but who are predisposed to atopic diseases. It aims at activities that will reduce the risk of developing $A D$ in the future.

In the 80 s of the last century, the so called hygiene hypothesis was formulated which was based on the observation that atopic diseases were less common in children growing up in families with many children [6]. It assumed that excessive attention to hygiene, changes in eating habits, the widespread use of antibiotics and immunization increased the risk of developing AD. It was even thought that this might be due to the decreased exposure to certain pathogens in infancy.

Numerous studies have confirmed this theory. German researchers observed a significantly increased risk of $A D$ in children from small families who began attending kindergarten late in their life. In the case of large families, the age of the child who was sent to the crèche or kindergarten was not relevant to the development of AD [7]. In two other studies, children who previously participated in activities in child care centres or crèches were at a significantly lower risk of developing AD [8, 9]. Other observations conducted by Alm et al. on the communities adhering to the anthropozoic philosophy (the movement founded by Rudolf Steiner in the early years of the twentieth century referring to many areas of life such as medicine and agriculture) have led to the conclusions that AD is much less common in this environment. This community strongly limited the use of antibiotics and immunization, and their diet was based largely on fermented vegetables containing Lactobacillus sp. [10].

Recently published studies, however, have not confirmed the hygiene hypothesis. Zutavern et al. as well as Purvis et al. in their observations did not show a positive correlation between AD and the size of the family [11, 12]. According to Hungarian authors, the fact whether the child attends a kindergarten or not, how often it suffers from infection or whether it is brought up at home does not affect the development of AD [13]. Moreover, the same authors together with Braun-Fahrlander et al. and Perkin and Strachan did not observe less frequent incidence of $A D$ in children living in rural areas than in those from large cities [14, 15].

\section{Infections}

The study on the impact of maternal infection during pregnancy and future development of $A D$ in children has shown that more than two infections significantly increase the risk of AD in children [16].

Most authors have focused their research on infections affecting children and their impact on the development of AD. Among others, the impact of mumps, pneumonia, diarrheal diseases, measles, and chickenpox was described. Most of the studies indicate an increased risk of $A D$ in children regularly exposed to infections $[17,18]$.

In the case of viral hepatitis, herpes simplex virus or Helicobacter pylori infections, no association was found between these diseases and the prevalence of AD. Nevertheless, in patients after $\mathrm{H}$. pylori eradication, the risk of AD was reduced by 30\% [19].

Lipopolysaccharide (endotoxin) forming a protein-lipid film on the surface of Gram-negative bacteria is known as an inducer of IL-10 and interferon $\gamma$ (IFN- $\gamma$ ). On this basis, a theory has been proposed which explains the effect of animals and the rural environment on reduction of the $\mathrm{AD}$ risk, due to the greater exposure to $\operatorname{Gram}(-)$ bacteria infection in this environment. In two studies, German and American ones, a negative correlation was observed between endotoxin and the risk of $A D$ in the first year of child's life. In the German study, the protective effect, however, disappeared in the second year of life [20, 21].

Gastrointestinal endoparasites are a strong promoter of Th2-dependent responses associated with the production of large amounts of IgE, which in theory should increase the risk of incidence of atopic diseases. The studies show, however, that the invasion of duodenal hookworm, human Ascaris, and Schistosoma haematobium protects against the development of asthma and atopy, and reduces the occurrence of positive skin tests to house dust mite (HDM). The explanation of this phenomenon can be related to the production of anti-inflammatory cytokines in the course of chronic helminthiasis [22, 23].

\section{Vaccination}

Vaccination is associated with increased exposure to infectious antigens, which can exacerbate the Th2 response and thus promote the development of atopic diseases. Adjuvants and vaccine stabilizers may also induce allergy. Faroogi and Hopkin have shown a slight increase in the risk of $A D$ in 12-year-old children after vaccination for whooping cough, tetanus and diphtheria [24]. In the Danish study, there was almost a twofold increase in the risk of developing AD in children aged 3-15 years after vaccination against measles, mumps and rubella [25]. In several other studies, no association between vaccination and an increased risk of the AD development was found $[26,27]$. However, in a recently published study, Martignon et al. [28] observed a significantly lower risk of asthma and atopic diseases in children vaccinated according to the vaccination schedule than in children who were not vaccinated. They did not notice significant 
differences between the type of vaccination, number of injections, and the development of AD. An increased protection was observed only in the case of live-attenuated oral poliovirus and the bacillus Calmette-Guerin (BCG) vaccine. The authors also indicated that vaccination may promote the Th1 response and reduce the risk of developing $A D$ via this mechanism.

\section{Tuberculosis, BCG}

In a number of studies, no relationship between tuberculosis, BCG vaccination and the development of atopic diseases has been stated [29-31]. Tuberculosis is a disease inducing Th1 response and might therefore contribute to reducing the incidence of $A D$. In the Japanese study, $50 \%$ lower risk of developing AD was reported in children aged 12-13 years with a strong response to tuberculin, which is associated with the promotion of Th1 response and the production of IFN- $\gamma$ [32].

\section{Antibiotics}

The study results on the effects of antibiotics in children and the risk of $A D$ often show a positive correlation [24, 33-35]. In two investigations, the association was not significant $[36,37]$. It is believed that the use of antibiotics can contribute to an increased risk of $A D$ since antibiotics destroy the intestinal flora, affecting in this way the immune system.

Results of research on the influence of Lactobacillus on the course of AD suggest that their administration significantly reduces the risk of $A D$ or its severity. In a randomized double-blind placebo-controlled test, $1 \times 10^{10}$ colonies of Lactobacillus rhamnosus vs placebo were administered to pregnant mothers with a history of atopy. They were given for 2-4 weeks before the birth to mothers and for 6 months after the birth to children or nursing mothers. Observations were made for 2 years. The prevalence of $A D$ in children who received these bacteria was 50\% lower compared to placebo [38, 39].

Perkin and Strachan found that children who drank unpasteurized milk, which contains probiotic bacteria, irrespective of the place of living, were at a much lower risk of developing AD. Consumption of such milk affected the reduction in total IgE levels in serum and correlated with a significant increase in the production of IFN- $\gamma$ [15].

\section{Breastfeeding}

The relationship between breastfeeding and the development of atopy and food allergy in early childhood is controversial. Exclusive breastfeeding of infants up to 6 months of age is recommended for prevention of atopic diseases. It is suggested that a low concentration of cow's milk proteins in human milk may induce tolerance to those proteins [40]. Also compounds like polyunsatu- rated fatty acids (PUFA) and cytokines from breast milk may affect the risk of allergy. Some authors observed that a small quantity of $\alpha$-linolenic acid and high proportion of n6/n3 fatty acid in breast milk increase the frequency of atopy [41, 42]. Many authors agree that breastfeeding up to 3 months of age reduces the risk of allergy or causes that its course is milder [43-45]. A study of 4089 children shows that breastfeeding for at least 4 months reduces the incidence of eczema up to 4 years of age [46]. Another study, in which children under the age of 17 were observed, revealed a close relationship between exclusive breastfeeding of infants up to 6 months of age and a lower risk of eczema in the first and third year of life. The authors also emphasize the beneficial effect of breastfeeding on the symptoms of respiratory allergies, as compared to children breastfed for less than 3 months [47]. Australian researchers based on their observations reported that the introduction of foods other than breast milk before the age of 4 months of age significantly increases the risk of asthma and atopy in children over 6 years of age [48].

In several other studies, the authors did not show any relationship between breastfeeding and a lower prevalence of atopic diseases $[49,50]$. Swedish researchers observed a group of 8300 children born between 1997 and 1999 for the relationship between the development of $A D$ and exclusive breastfeeding. They stated that the duration of breastfeeding did not affect the development of AD [51].

There are also studies in the available literature that describe an adverse effect of breastfeeding on the development of allergic diseases. Bergmann et al. [52] demonstrated that several-month breastfeeding of infants whose parents suffer from allergic diseases increases the risk of atopic eczema, however more often when the mother is genetically loaded. Moreover, the results from New Zealand indicate the adverse effect of exclusive breastfeeding up to 6 months of age on the risk of developing AD [12]. Breastfeeding may predispose to development of allergy to cow's milk and the other proteins in susceptible children. This is a result of ingestion of low doses of the antigen especially when mother's milk contains low amounts of IgA and transforming growth factor $\beta$ (TGF- $\beta$ ) [53].

Whether breastfeeding has a protective influence against atopy remains controversial. It is due to the fact that we cannot conduct randomized double-blind studies for ethical reasons.

Breastfeeding can also worsen the skin condition in children with severe atopic eczema, especially in IgEmediated food allergy. This is a result of presence of food allergens in mother's milk who does not follow a restriction diet. More than $60 \%$ of children in Heine et al.'s study present IgE sensitization (FS-IgE) to products which had not been ingested directly. What is more, in 18\% of chil- 
dren an acute allergy reaction after first exposure to cow's milk, eggs and nuts occurs [54]. It suggests that FS-IgE is acquired during pregnancy - transplacentally or during breastfeeding. It could explain the fact that some studies show that restrictive diet without milk, eggs or fish during lactation have a protective effect on children against atopy $[55,56]$. However, the majority of authors do not confirm that the special diet during breastfeeding and pregnancy may decrease frequency of food allergy [57-59]. Several prospective studies show a preventive effect on development of $A D$ in high-risk infants fed with extensively hydrolysed formulas (eHF) with avoidance of cow's milk proteins and solid food until 4 months of age. Nevertheless, eHF in infants older than 6 months who were fed with breast milk previously do not protect them against atopy better than cow's milk formulas (CMF). There is no evidence of preventing the development of allergy by restrictive diet after 6 months of age. Studies do not show any difference in allergy development between eHF or breast milk ingestion for the first 4-6 and 12-24 months [60-63].

\section{Animals}

Many authors believe that the possession of fur animals in the house positively affects the development of the child's immune system and may have a protective impact on the development of atopic diseases. The fact that parents with a history of atopic diseases often avoid animals may pose a problem in the interpretation of the study results. According to Ludvigsson [63], keeping fur animals in the house significantly reduces the incidence of $A D$ in children and is not related with the occurrence of atopic tendency in the family. Schoetzau et al. drew similar conclusions from their research [64]. The mucosal immune system of newborns produces specific molecules against intestinal bacteria which may contribute to the response to other antigens such as animal and food allergens. It is thought that early exposure to animal allergens plays a protective role in this process. Several studies suggest that the immune response to animal allergens appears already in intrauterine life. Furthermore, high concentrations of IgG antibodies directed against animal allergens are associated with a lower prevalence of atopic changes up to 8 years of age. Purvis et al. in their research [12] estimated that keeping cats by children aged 3-5 years has a negative correlation with the development of AD. According to another author, exposure to more than one cat in the $1^{\text {st }}$ year of life may reduce the risk of sensitization to the allergen panel but not to cats [65]. However, having a dog prevents the development of asthma symptoms but does not reduce sensitization to dogs [66]. Epidemiological studies suggest that the prevalence of sensitization to dogs is lower in the environment with low levels of allergens [67]. Some authors even believe that having pets can predispose to the development of AD [68].

\section{Apartment renovation}

Wen et al. in their study in Taiwan [69] reported that children born to mothers who during pregnancy had been housed in renovated areas were more likely to develop AD. Wall paints, floor varnishes and new furniture secrete chemical substances such as pigments, monomers, solvents and surfactants. Many of them have been found to promote the development of allergies. Volatile organic components pass through the placenta to the foetus and lead to increased production of IL-4 and IFN- $\gamma$ decrease. In addition, they destroy the epidermal barrier function and enhance the adverse effect of house dust mite (HDM) on patients with allergy to dust mite.

\section{Fungi}

In the same study, the author described a significant relationship between the risk of developing asthma, atopy and respiratory tract symptoms, and the presence of Aspergillus and Penicillium fungi in the place of residence. The larger the number of occupied walls, the higher the risk is. In this case, specific conditions in Taiwan, where the average temperature is $15-30^{\circ} \mathrm{C}$ and humidity is $70-80 \%$, favour the fungal growth on walls. In our climatic conditions, this is probably not such an important issue.

The beneficial methods which can protect against development of $A D$ are:

- breastfeeding or ingestion eHF in the first 4-6 months of age,

- introduction of solid food after 4-6 months of age,

- administration of probiotic bacteria in pregnant women 2-4 weeks before delivery and in infants for 6 months after birth,

- reduction of administration of antibiotics in infants,

- avoiding houses under renovation during pregnancy,

- avoiding infections in infants.

\section{Secondary prevention}

Secondary prevention refers to subjects with diagnosed $A D$ and consists of measures aimed at preventing the appearance of skin lesions as well as reducing the use of strong pharmacotherapy.

\section{Epidermal barrier}

The stratum corneum - the outermost layer of the epidermis - creates the barrier against the penetration of allergens and irritants. The presence of the intercellular components called corneodesmosomes ensures the integrity of this layer. Corneocytes that make up the stratum corneum are flattened cells devoid of nuclei with densely packed keratin fibres, which constitute the final stage of keratinocyte differentiation. During the formation of the corneocytes, granule cells secrete granules in 
the form of lamellar bodies into the extracellular space. Secretion includes lipid components such as cholesterol, ceramide precursors, free fatty acids and enzymes needed for their formation. They fill the intercellular space forming a bilayer lipid matrix and a water-fat coat along with sebum and sweat on the skin surface. Lipids derived from lamellar bodies prevent transepidermal water loss (TEWL) and the penetration of hydrophilic substances. Moreover, lamellar bodies provide the stratum corneum with proteases and their inhibitors nourishing corneodesmosomes. Disorders of maturation and lamellar body delivery have been documented in patients with AD. This results in a reduced amount of fatty acids, lipids and enzymes of the stratum corneum, leading to the damage to the epidermal barrier functioning.

During differentiation of keratinocytes their membrane is converted to the insoluble horny plate, a layer called a cornified envelope, which gives strength and forms a scaffold for lipid binding. The cornified envelope is composed of structural proteins, loricrin, involucrin, filaggrin, and small proline-type peptides. Filaggrin is especially important because it aggregates collagen fibres of the cytoskeletal cells into bundles, thus flattening the corneocyte into the disc of a large outer surface. In addition, filaggrin is degraded to short peptide chains and free amino acids, which are components of the natural moisturizing factor (NMF), which includes, among others, lactic acid, carboxylic acid, pyrrolidone carboxylic acid, procaine acid and urea. The NMF is essential for water retention within the corneocyte, which allows to maintain optimal hydration. Pyrrolidone carboxylic acid and lactic acid are humectants, which are compounds with strong hygroscopic action. They prevent the formation of gaps between corneocytes and maintain the integrity of the stratum corneum, making it resistant to penetration of allergens. Furthermore, acids derived from NMF can produce acidic skin pH (5.4-5.9), which helps to maintain the proper barrier function, being involved in the process of exfoliation, warrants appropriate permeability and proper adhesion, as well as exerts an antimicrobial effect and favours skin colonization by non-pathogenic bacteria. Deeper layers of the skin have a higher $\mathrm{pH}$. This is associated with activity of enzymes that work under certain conditions. Serine proteases involved in the process of exfoliation need neutral $\mathrm{pH}$, and cathepsin, $\beta$-glucocerebrosidase and acidic sphingomyelinase require acidic environment. PH gradient along the epidermis is very important in regulating shedding and generating lamellar bodies.

In patients with $\mathrm{AD}$, a mutation in the gene encoding prophilagrin, the precursor of filaggrin, can be observed, which contributes to the reduction in the filaggrin and NMF level in the skin of these patients. This leads to a decrease in water holding capacity due to the reduced amount of humectants and the impairment in the cornified envelope structure and the reduction in elasticity and mechanical resistance. The barrier defect increases the risk of penetration of irritants and allergens. In addition, there is a significant increase in $\mathrm{pH}$ of the stratum corneum of the healthy skin and that with eczematous lesions. The increase in $\mathrm{pH}$ is also manifested in increased activity of serine proteases and their higher concentration in the stratum corneum. Moreover, $\mathrm{pH}$ increase reduces the activity of cathepsin, $\beta$-glucocerebrosidase and sphingomyelinase, which require an acidic environment contributing to ceramide decrease. It is suggested that their activity is an important indicator of damage to the barrier. Activity of trypsin, tryptase, plasmin, and urokinase (they activate the PAR2 receptor through which inflammatory response and itching sensation are mediated) positively correlates with transepidermal water loss, and inversely with the hydration of the epidermis layers. Serine proteases and their inhibitors are involved in the process of the stratum corneum desquamation by reducing corneodesmosome adhesion. Corneocytes peeled from the surface of the skin are constantly replaced by the epidermal cells that have completed the process of terminal keratinization. Peeling provides the balance to the skin barrier and allows to continue the epidermal cell regeneration.

Clinical studies have shown that the filaggrin gene mutation results in skin dryness, roughness and sensitivity. There is a positive correlation between the severity of the disease and the impairment in the skin barrier [70].

\section{Protection of the skin barrier}

In order to restore the barrier function in patients with $A D$, prevention measures focused on avoiding contact with irritants, especially exsiccating ones should be used together with regular and proper care.

Atopic dermatitis patients should avoid using soap and detergents, which are one of the most common causes of skin irritation. Detergents function through emulsification of surface lipids both natural and of external origin, which are then washed with water. The negative effect of a surfactant on the skin barrier is reflected in an increased TEWL, which is intensified in patients with AD. Washing the skin with soap contributes to the $\mathrm{pH}$ increase for at least $90 \mathrm{~min}$. This impairs the barrier function in the protease-dependent mechanism. The irritating effect of soap and detergents can be partly explained by an increased release of proinflammatory cytokines from the corneocytes. The use of detergents additionally interferes with the expression of key markers of keratinocyte and proteolytic enzyme differentiation. Cosmetics, containing various preservatives, dyes and fragrances, are, apart from soap and detergents, substances that can cause irritation. Therefore, the best way is to use products with fewer ingredients which do not contain any preservatives and are colourless and odourless. 
New clothes contain high levels of formaldehyde, which can also irritate the skin. Thus, it is advisable to always wash all new clothes before use. Washing powders can also be a source of irritation, especially when they remain on clothes after laundering. Washing gels, free of phosphates and extra rinses are recommended. However, we do not recommend the use of bleaching or softening agents.

The environment that surrounds us is important in $A D$ prevention. The air temperature should be about $18-22^{\circ} \mathrm{C}$. In summer, subjects susceptible to AD should stay in air-conditioned rooms, which should be heated in winter. Frequent and sudden changes in temperature are not beneficial. Air should be adequately humidified and appropriate air humidifiers should be used in winter. Frequent baths are not advisable, especially in very hot water. Fluorescent light and excessive exposure to UV radiation should be avoided. The use of suitable protective SPF filters is recommended. Moreover, topical corticosteroids, so often used with great success in $A D$, lead to the thinning of the skin and reduction in the amount of intercellular lipids resulting in increased TEWL. This favours the recurrence of eczema lesions after discontinuation of topical corticosteroids.

Emollients are the basic substances used in skin care of patients with $A D$, which affect the recovery of the impaired skin barrier. They are based on a biologically inert substrate presenting two types of particles - oil-in-water (O/W) or water-in-oil (W/O). Lipids used in the production of emollients are animal fats, vegetable oils, waxes, cocoa butter, and mineral hydrocarbons. Enriching substances which supplement lipid substances in the skin, such as ceramides, phospholipids, waxes, squalenes, cholesterol and free fatty acids are also added. Emollients work in two ways: via hydration of the epidermal stratum corneum due to the presence of water-binding ingredients as well as via reduction in the TEWL due to the occlusal action. The components which penetrate the epidermis and bind water are the following: urea, pyrrolidone carboxylic acid, glycerol, hyaluronic acid and lactic acid. Lipids, animal fats, vegetable oils and waxes as well as fruit acids are the factors which seal the epidermal barrier. Some emollient components also have anti-mitotic, anti-itching and anti-inflammatory activity owing to the inhibition of the prostanoid production, which affects cyclooxygenase. Emollient action lasts up to $6 \mathrm{~h}$ so it is important to repeat its application and to use the agent regularly $[71,72]$.

\section{Bacterial superinfections}

The skin of AD patients is susceptible to colonization by Gram-positive Staphylococcus aureus, which is found in up to $90 \%$ of patients. Both the inflammatory microenvironment and the defective epidermal barrier are characterized by the increased permeability, which predisposes the skin to colonization by this pathogen. The increased surface $\mathrm{pH}$ and reduced levels of higher fatty acids (HFA), ceramide metabolites, and sphingosine, which have antimicrobial properties, make the skin more likely to be colonized by Staphylococcus aureus. In addition, antimicrobial proteins, the product of human cathelicidin LL-37 (hCAP), and the human $\beta$-defensin 2 and 3 (hBD2, hBD3) are reduced in the Th2-dependent mechanism. LL-37 is essential to maintain the integrity of the epidermis, and hCAP and hBD3 play an important anti-Staphylococcus role.

Staphylococcus aureus alone adversely affects the barrier function, worsening its defect. Surface proteins of $S$. aureus reduce HFA production and increase ceramidase production. At the further stage of the disease, nontoxigenic Staphylococcus strains are replaced by strains generating enterotoxin, which is likely to promote and maintain inflammation giving rise to a further cause of itching. Staphylococcal enterotoxin can act as a superantigen stimulating proliferation of T and B cells [73]. T lymphocytes activated by enterotoxin produce significantly more IL-31 in patients with AD as compared to the control group. Interleukin-31 is one of the mediators which induces inflammation and itching in AD patients [74]. Enterotoxin also increases the expression of a glucocorticosteroid-induced protein ligand, thus inhibiting activity of regulatory cells, and evokes a beta receptor isoform for glucocorticosteroids in mononuclear cells, which favours the formation of resistance to glucocorticosteroids [1].

Adequate skin care that restores proper functioning of the skin barrier plays an important role in prevention of $S$. aureus infections. Among the newly developed therapies preventing bacterial colonization of the skin, the probiotic bacterium, $S$. epidermidis, is mentioned, which affects the growth of $\beta$-defensin 2 and 3 production through the increased expression of toll-like receptor 2 (TLR2). This provides promising results in prevention of this pathogen colonization [75]. In addition, ribonuclease, RNase 7, usually occurring in the human skin, exhibits the antimicrobial activity. Its expression is induced by $\mathrm{IL}-1 \beta$ and IFN- $\gamma$. RNase 7 is quickly produced in the skin (within $2 \mathrm{~h}$ ) after the penetration of staphylococci, which effectively inhibits further colonization [76].

\section{Importance of food allergy}

Food allergy usually afflicts children up to the age of 2 years but it is not so important in adults. Food allergens may contribute to triggering and exacerbating AD. It has not been established until now whether this takes place due to the allergic or non-immune mechanism. Food allergy occurs in approximately $30 \%$ of people with a severe or moderate form of $A D$, which is confirmed by double-blind placebo-controlled trials. Eggs, milk, wheat, soy, peanuts, hazel nuts, and fish are the most common food allergens. Diagnosis of food allergy should begin with 
a thorough history, and only then allergy tests can be performed. Elimination diet is not recommended when the probability of food allergy confirmed by the tests is not high because this diet may lead to patients' malnutrition and poor quality of life. However, if allergy tests indicate a high probability of the allergic factor triggering allergic symptoms of AD, the elimination diet should be used for 4-6 weeks. When the test results are inconclusive, the diet low in allergenic food products should be used for 3 weeks. Infants are fed highly hydrolyzed cow's milk or amino-acid derived formulas. An oral provocation test is however the gold standard for the diagnosis of food allergy. If allergy symptoms persist for 4 weeks of using the elimination diet, food allergy can most likely be excluded as a cause of allergy symptoms. If at this time, a significant improvement has been observed, there is a good chance that food products are the cause of the ailments. Treatment is based on the diet eliminating allergenic types of food. As for infants who are breastfed often the restrictive diet of the mother is recommended. The antigens against cow's milk, eggs, wheat, nuts were detected in breast milk within 2-4 h after ingestion. That is why infants suffering from atopic eczema with cow's milk allergy may develop the skin lesion several hours after maternal ingestion of cow's milk products [77, 78]. Sometimes the breastfeeding or cow's milk formulas can provoke the skin lesion in children with severe AD. In this case, the cessation of breastfeeding or cow's milk formulas and administration of hydrolysed formulas greatly improves the condition of the skin and is recommended. It is very important to educate patients and their families as to the proper reading of labels on food products: they should not contain even traces of sensitising food. Food allergy will subside in one-third of children within 3 years, on condition that the recommended diet is strictly observed, which inhibits hypersensitivity to a particular nutritional component in the future. Whether food allergy subsides and how quickly it happens largely depends on the type of sensitizing food. Food allergy should quickly disappear when the sensitization has been caused by egg white, milk or wheat. If a child is allergic to nuts, fish or seafood, the symptoms associated with the consumption of these products may persist even for the whole life. Allergy should be controlled every 12-18 months. The measurement of food-specific IgEs may also be helpful in determining the prognosis. The lower their level, the greater the chance for faster relief of allergy is $[79,80]$.

\section{Role of inhalant allergens}

After the third year of life, the prevalence of food allergies decreases, however, that of inhalant allergies increases. The concentration of IgE to inhalant allergens increases with age. Sensitization to pollen is observed in 3-4 year-old children [81] but it can occur even during the first month of age [82]. The results of Swedish authors show that excessive exposure to birch-pollen allergens in early infancy increases the risk of birch-pollen allergy and pollen induced asthma [83]. A study of Breuer et al. proves that children with $A D$ and birch-pollen allergy more often develop late eczematous reaction after ingestion of birch-pollen associated food [84]. Patients with pollen allergy often develop IgE response to crossreactive food allergens. Plants which induce sensitization come from Rosaceae, Solanaceae and Umbelliferae family. We distinguish apples, hazelnuts, carrots and celeries which most often cause allergy [85]. It is proved that birch pollen-related food may lead to deterioration of the skin condition [86]. When dealing with moderate to severe $A D$, positive test reactions to mites, mould, yeast and animals can be more frequently observed. Their role in the pathogenesis of AD has not been well understood. Contact with inhaled allergens may lead to eczema. The skin contact with these allergens may cause inflammation. Specific IgEs against house dust mites are detected in $95 \%$ of patients with AD and only in $42 \%$ of those with asthma. A medical history, skin prick tests (SPT) and specific IgE measurements are helpful in the diagnosis of AD. Atopic patch tests (APT) are also used as a diagnostic tool to assess skin reactions to different inhaled allergens. APTs have higher specificity than skin prick tests and measurements of specific IgE but lower sensitivity. The indications for performing APT are the following: a suspicion of inhalant allergy due to negative results of SPT and specific IgE measurements, severe and refractory $A D$, the lack of a known causative factor, and the presence of hypersensitivity to a variety of allergens when the relationship between the disease and any of the above factors has not been clinically confirmed. In the treatment and prevention of $\mathrm{AD}$, the most important thing is to avoid the allergen. The most common allergens are house dust mites (HDM) that develop in the warm and humid environment [79]. It is therefore necessary to maintain the room humidity within $40-50 \%$ and the temperature of $18-19^{\circ} \mathrm{C}$. Frequent airing the rooms and the use of vacuum cleaners with special anti-allergic filters are recommended. Eiderdowns and feather pillows should be removed and replaced with anti-allergic bedding. The mattresses should have special covers; carpets, curtains and upholstered furniture, which are favourable dust mite habitats, should be removed. In the case of sensitization to another inhaled allergen, an attempt should be made to eliminate it from the surrounding environment.

\section{Pruritus/itching}

Itching, one of the primary symptoms of $A D$, is the criterion for the diagnosis of this disease and adversely affects the quality of patient's life. As it is most pronounced during the night, it results in sleep disorders. It also contributes to psychosocial disorders such as 
depression, agitation, anxiety, changes in eating habits, decreased self-esteem, and difficulties in concentration. Itching leads to scratching which further causes destruction of the epidermal barrier function, inducing inflammatory symptoms. Pruritus pathogenesis is not well understood. It is known that it involves mechanisms of the peripheral and central nervous system as well as mediators associated with the formation of pruritus. Proteases present in excessive amounts in the epidermis of AD patients play an important role in mediating itching through activation of the PAR2 receptor, whose expression is increased in epidermal nerve fibres, keratinocytes and the endothelium in AD patients. Additionally, the concentration of mast cell tryptase, an agonist of PAR2, is significantly increased, whereas the concentration of histamine is normal. Histamine-sensitive afferent nerve fibres that convey the feeling of itching have been identified among the human peripheral nerve fibres. Similar nerve fibres that connect the dorsal horns with the thalamus have been also detected in cats. This suggests the existence of a specialized neural path for itching sensation. On the other hand, even antihistamines cannot eliminate large quantities of pruritus in patients with $A D$, which means that histamine is not a major mediator of itching. Moreover, proteinase activation increases in staphylococcal infections and allergic skin diseases, which aggravates the course of AD. Mediators secreted by keratinocytes such as opioids, proteases, substance P, neurotrophin, acetylquoline and endocannabinoids have been found to be involved in formation of itching.

Positron emission tomography and magnetic resonance imaging show the areas of the cerebral cortex receiving itching sensation, which are also involved in the process of experiencing pain. Studies have demonstrated that these areas are different in healthy subjects and patients with $A D$. In the case of healthy individuals, the areas receiving itching sensation are located only within the sensory cortex, and in the case of AD patients, the structures of the brain responsible for receiving emotion are also involved in this process [87].

Factors such as sweating, dry skin, physical effort, emotional stress, wool or synthetic fibres, hot water, and certain foods can aggravate itching. A simple way to control the itchy skin is its cooling through properly selected clothes to match the ambient temperature. The adequate care involves wearing bright, cotton, loose-fitting clothes. Direct skin contact with wool, silk and flannel should be avoided along with overheating, excessive heat and excessive humidity. Temperature and humidity should be selected so as not to make the patient sweat. The sweat additionally irritates the skin of the AD patient, which increases itching. Staying in air-conditioned rooms is recommended in summer. Relatively normal everyday activities including sport should be maintained. Swimming seems to be the most recommended physical activity. Patients do not sweat during effort and their skin is not irritated by clothing. They should remember about taking a shower immediately after leaving the chlorinated water. Moderate exposure to the sun is also recommended. Patients should use creams with UV filters. In many AD patients, UV radiation reduces skin lesions, however longer exposure can result in significant skin irritation. The measures used to control dry skin can also, to a large degree, help to reduce the itching sensation. First of all it is advisable to use emollients regularly.

\section{Stress}

Recent studies show an important role of stress in the evolution of $A D$ and highlight the relationship between psychological factors and elements of the neurological and immune systems. Atopic dermatitis is characterized by lesions in the immune system with a strong response to non-specific factors, which is mediated by Th2 cell line activating $B$ cells to produce immunoglobulins. It appears that polarization towards the Th2 response occurs in early childhood and may be associated with stress. The number of eosinophils and the IgE expression increase in response to stress-generating factors. Stimulation of $T$ cells in response to stress takes place in several mechanisms. Stress hormones exert an immunosuppressive effect mainly directed towards the Th1 cells, thus resulting in the dominance of the Th2 response. Another pathway to stimulate the immune system cells leads through the fibres of the nervous system. The neuro-immune signal transduction involves neuropeptides and neurohormones. The crucial combination of the nervous and immune system is the interaction between nerve fibres and the mast cell. Mast cell degranulation takes place due to the stimulation by various substances secreted into the nerve endings including SP and calcitonin generelated peptide (CGRP). The number of connections between the nerve cells and mast cells is elevated in AD patients, both in the healthy and pathologically changed skin. Reduced reactivity of the hypothalamic-pituitaryadrenal axis, which may be due to the reduced expression or function of receptors for glucocorticoids can be observed in the course of stress. Children of mothers who during pregnancy were subject to prolonged stress have an increased cortisol secretion in response to stress, and the reduced ratio of Th1/Th2 cells. Stress occurring in childhood causes sensitization of the hypothalamicpituitary-adrenal axis, which increases susceptibility to stress in adulthood. A similar response is observed in patients with AD. Excessive stimulation of the hypothalamic-pituitary-adrenal axis in response to collecting a blood sample from the heel was observed in newborns with elevated IgE levels or a positive family history of atopy. It is most likely that corticotrophin-releasing hormone $(\mathrm{CRH})$ is involved in the pathogenesis of the above lesions. Increased activity of $\mathrm{CRH}$ is a persisting consequence of early stress. Epidermal cells of hair follicles produce $\mathrm{CRH}$, 
which locally stimulates mast cells to degranulation. In addition, the skin cells produce proopiomelanocortin and its products: $\alpha-M S H$, adrenocorticotropic hormone and $\beta$-endorphins. Thus, the skin secretes the same hormones which are secreted on the hypothalamuspituitary-adrenal pathway reflecting the phenomena in the peripheral nervous system. The local production of glucocorticosteroids in the skin is also possible due to the presence of all the necessary components. Some proteins such as proopiomelanocortins have the suppressor potential. $\alpha$-MSH inhibits IL-1, -2, -6, tumor necrosis factor $\alpha$ (TNF- $\alpha$ ), and adhesion molecules and increases the level of IL-10, whose dermal application as well as intracerebral injection inhibit a delayed-type hypersensitivity reaction. In addition, stress adversely affects the integrity and adhesion of the stratum corneum which may result from increased blood levels of glucocorticosteroids.

Based on many reports highlighting the role of stress in $A D$, it is thought that an attempt should be made to reduce the level of stress, which can decrease severity of skin lesions. There are many types of therapies aimed at reducing perceived stress including psychotherapy, relaxation therapy, hypnosis, massage or acupuncture. Psychological treatment should help to relieve anxiety, improve response to frustration and change the patterns of itching-scratching. Cognitive behavioural therapy is used to gain insight into the problems associated with $A D$ as well as to change behavioural patterns. It involves a positive conversation with oneself, replacing a negative one, and presents new opportunities to change the thought patterns of the patient. Destructive behaviour such as scratching should be replaced with less harmful action, which should be repeated for a long time until it becomes a conditional action. Education and parents' insight into their emotions, which can alter their behaviour towards the sick child, play an important role. The results of the use of autogenous training, cognitive-behavioural therapy, and educational program translate into reduced doses of corticosteroids as compared to AD patients treated only with the standard method.

Various forms of relaxation therapy, biofeedback autologous training, massage or hypnosis, are recommended. They are designed to reduce the level of anxiety and stress, but the results achieved in controlled studies in AD are often unsatisfactory. Biofeedback can be useful in diseases with an autonomous component, e.g. when there is a predominance of the sympathetic system in AD patients. Patients learn how to establish new patterns of behaviour by a conscious change in autonomic responses. Hypnosis can intensify the effects of biofeedback and support other treatments. Hypnosis is a condition in which the patient accepts the suggestions of the therapist, facilitating the creation of the new reality. The mechanism through which hypnosis improves skin conditions in dermatological disorders has not been completely elucidated. It is possible that, as in the case of biofeedback, the autonomous nervous system is involved. In an uncontrolled study with the use of hypnosis in the treatment of $A D$, the majority of patients showed significant improvement associated with reduced sensation of itching as well improvement in sleep and mood. Autogenic training is a form of self-hypnosis. The review of research on the use of this form of therapy demonstrates that the therapy results are comparable to those achieved with the use of biofeedback. Massage is another method used as a stress-reducing treatment. Children whose parents performed a 20-min massage once a day for a month showed a lower level of anxiety and their clinical symptoms improved.

A large meta-analysis reported that the best results are achieved through the use of autogenous training and cognitive-behavioural therapy, and the most effective psychological treatment is related to a combination of techniques to cope with stress, relaxation and behavioural changes. The education program can maintain therapeutic results of these methods [88].

The most important recommendations in secondary prevention are:

- application of emollients every 4-6 h,

- elimination of skin irritants,

- elimination of food allergens in diet,

- avoidance of sweating,

- avoidance of stress situations or stress-reducing treatment.

Primary prevention of $A D$ in many cases is controversial. The hygiene hypothesis formulated in the 1980s, in which the cause of higher prevalence of $A D$ is associated with a less frequent contact with pathogenic microorganisms related to increased attention to hygiene, the use of vaccination, and less frequent contact with animals, does not seem nowadays to have such a significance as it has been thought. However, the intestinal flora, which modulates the immune system inducing tolerance to antigens derived from the outside might play an important role. Accordingly, the use of antibiotics, which destroy microorganisms of the gastrointestinal tract may contribute to the increased risk of AD development. On the other hand, products containing probiotic bacteria may protect against the disease. Although some studies have shown that exclusive breastfeeding until the child is 6 months old prevents atopic diseases, the obvious evidence that prolonged breastfeeding reduces the risk of $A D$ is still missing.

Adherence to secondary prevention of AD is however of great importance as it often allows to completely discontinue drug therapy. Secondary prevention is mainly focused on the care of the skin, in which the use of emollients and avoidance of irritants or sensitizers play a fundamental role. Therefore, it is extremely important to educate patients and their families which substances they should avoid and how they should adjust the patients' conditions of life not to trigger the disease re- 
lapse. A medical history of the patient and their families is the beginning of diagnosis for exclusion of allergenic factors from the environment. Furthermore, psychotherapy aimed at reducing the level of stress, which also contributes to the exacerbation of $A D$, may be very helpful in controlling atopic diseases.

\section{Conflict of interest}

The authors declare no conflict of interest.

\section{References}

1. Bieber T. Atopic dermatitis. N Eng J Med 2008; 358: 1483-94.

2. Bergmann RL, Edenharter G, Bergmann KE, et al. Predictability of early atopy by cord blood-lgE and parental history. Clin Exp Allergy 1997; 27: 752-60.

3. Nilsson L. Risk factors for atopic disease in childhood (Linkoping University Medica Dissertation no 556). Linkoping University, 1998.

4. Ruiz RG, Kemedy DM, Price JF. Higher risk of infant ile atopic dermatitis from maternal atopy than from paternal atopy. Clin Exp Allergy 1992; 22: 762-6.

5. Hjern A, Haglund B, Hedlin G. Ethnicity, childhood environment and atopic disorder. Clin Exp Allergy 2000; 30: 521-8.

6. Strachan DP. Hayfewer, hygiene and household size. BM 1989; 299: 1259-60.

7. Kamer U, Heinrich J, Wjst M, Wichmann HE. Age of entry to day nursery and allergy in later childhood. Lancet 1999 353: 450-4.

8. Celedon JC, Wright RJ, Litonjua AA, et al. Day care attendance in early life, maternal history of asthma, and asthma at the age of 6 years. Am J Resp Crit Care Med 2003; 167: 1239-43.

9. Benn CS, Melbye M, Wohlfahrt J, et al. Cohort study of sibling effect, infectious diseases, and risk of atopic dermatitis during first 18 months of life. BMJ 2004; 328: 122-30.

10. Alm JS, Swartz J, Lilja G, et al. Atopy in children of families with an antroposophic lifestyle. Lancet 1999; 353: 1485-8.

11. Zutavern A, Hirsch T, Leupold W, et al. Atopic dermatitis, extrinsic atopic dermatitis and the hygiene hipothesis: results from a cross-sectional study. Clin Exp Dermatol 2005; 35: 1301-8.

12. Purvis DJ, Thompson JMD, Clark PM, et al. Risk factors for atopic dermatitis in New Zealand children at 3-5 years of age. Br J Dermatol 2005; 152: 742-3.

13. Sebok B, Schneider I, Harangi F. Familiar and environmental factors influencing atopic dermatitis in the childhood. J Eur Acad Dermatol Venereol 2006; 20: 418-22.

14. Braun-Fahrlander CH, Gassner M, Grize L, et al. Prevalence of hayfever and allergic sensitization in farmers children and their peers living in the same rural community. Clin Exp Allergy 1999; 29: 28-34.

15. Perkin MR, Strachan DP. Which aspects of the farming life style explain the inverse association with childhood allergy? J Allergy Clin Immunol 2006; 117: 1374-81.

16. McKeever TM, Lewis SA, Smith C, Hubbard R. The importance of prenatal exposure on the development of allergic disease: a British cohort study using the West Midlands general practice database. Am J Resp Crit Care Med 2002; 166: 827-32.

17. Benn CS, Melbye M, Wohlfahrt J, et al. Cohort study of sibling effect, infectious diseases, and risk of atopic dermatitis during first 18 months of life. BMJ 2004; 328: 122-30.
18. McKeever TM, Lewis SA, Smith C, et al. Early exposure to infections and antibiotics and the incidence of allergic disease: a birth cohort study with the West Midlands general practice research database. J Allergy Clin Immunol 2002; 109: 43-50.

19. Uter W, Stock C, Pfahlerg A, et al. Association between infections and signs and symptoms of atopic hypersensitivity - results of a cross-sectional survey among first-year University students in Germany and Spain. Allergy 2003; 58: 580-4.

20. Liu AH. Endotoxin exposure in allergy and asthma: reconciling a paradox. J Allergy Clin Immunol 2002; 109: 379-92.

21. Braun-Fahrlander CH, Riedler J, Herz U, et al. Allergy and endotoxin study team. Environmental exposure to endotoxin and its relation to asthma I school-age children. N Eng I Med 2002; 347: 869-77.

22. Van den Biggelaar AH, van Ree R, Rodriguez LC, et al. Decreased atopy in children infected with Schistosoma haematobium: a role for parasite-induced IL-10. Lancet 2000; 356: 1723-27.

23. Yazdanbakhsh M, Kremsner PG, van Ree R. Allergy, parasites and the hygiene hipothesis. Science 2002; 296: 490-4.

24. Faroogi IS, Hopkin JM. Early childhood infection and atopic disorder. Thorax 1998; 53: 927-32.

25. Olesen AB, Juul S, Thestrup-Pedersen K. Atopic dermatitis is increased following vaccination for measles, mumps, and rubella infection. Acta Derm Venereol 2003; 83: 445-50.

26. Anderson HR, Poloniecki JD, Strachan DP, et al. Immunization and symptoms of atopic disease in children: results from the international study of asthma and allergies in childhood. Am J Public Health 2001; 91: 1126-9.

27. Kempt T, Pearce N, Fitzharris $P$, et al. Is infant immunization a risk factor for childhood asthma and allergy? Epidemiology 1997; 8: 678-80

28. Martignon G, Oryszczyn M-P, Annesi-Maesano I. Does childhood immunization against infectious diseases protect from the development of atopic disease? Pediatr Allergy Immunol 2005; 16: 193-200.

29. Wong Gw, Hui DS, Tam CM, et al. Asthma, atopy and tuberculin responses in Chinese schoolchildren in Honk Kong. Thorax 2001; 56: 770-3.

30. Von Mutius E, Pearce N, Beasley R, et al. International patterns of tuberculosis and the prevalence of symptoms of asthma, rhinitis, and eczema. Thorax 2000; 55: 449-53.

31. Yilmaz M, Bingol G, Altintas D, Kendirli SG. Correlation between atopic diseases and tuberkulin responses. Allergy 2000; 55: 664-7.

32. Shirakawa T, Enomoto T, Simazu S, Hopkin JM. The inverse association between tuberkulin responses and atopic disorder. Science 1997; 175: 77-9.

33. McKeever TM, Lewis SA, Smith C, et al. Early exposure to infections and antibiotics and the incidence of allergic disease: a birth cohort study with the West Midlands general practice research database. J Allergy Clin Immunol 2002; 109: 43-50

34. Von Mutius E, Illi S, Hirsch T, et al. Frequency of infections and risk of asthma, atopy and airway hyperresponsiveness in children. Eur Respir J 1999; 14: 4-11.

35. Droste JHJ, Wierenga MH, Weyler JJ, et al. Does the use of antibiotics in early childhood increase the risk of asthma and allergic disease? Clin Exp Allergy 2000; 30: 1547-53.

36. Wickens K, Pearce N, Crane J, Beasley R. Antibiotic use in early childhood and the development of asthma. Clin Exp Allergy 1999; 29: 766-71. 
37. Celedon JC, Litonjua AA, Ryan L, et al. Lack of association between antibiotic use in first year of life and asthma, allergic rhinitis, or eczema at age 5 years. Am J Respir Crit Care Med 2002; 166: 72-5.

38. Kalliomaki M, Slaminen S, Arvilommi H, et al. Probiotics in primary prevention of atopic disease: a randomised placebo-controlled trial. Lancet 2001; 357: 1076-9.

39. Kalliomaki M, Salminen S, Poussa T, et al. Probiotics and prevention of atopic disease: 4-year follow-up of a randomised placebo-controlled trial. Lancet 2003; 361: 1869-71.

40. Host A, Husby S, Osterballe O. A prospective study of cow's milk allergy in exclusively breast-fed infants. Acta Paediatr Scand 1988; 77: 663-70.

41. Duchen K, Yo G, Bjorkstein B. Atopic sensitization during the first year of life in relation to long chain polyunsaturated fatty acid levels in human milk. Pediatr Res 1998; 44: 478-84.

42. Duchen K, Yo G, Bjorkstein B. Polyunsaturated fatty acid in breast milk in relation to atopy in the mother and her child. Int Arch Allergy Immunol 1999; 118: 321-3.

43. Eigenmann PA. Breast-feeding and atopic eczema dermatitis syndrome: protective or harmful? Allergy 2004; 59 (Suppl. 78): 42-4.

44. Gdalevich M, Mimouni D, David M, et al. Breast-feeding and the onset of atopic dermatitis in childhood: a systematic review and meta-analysis of prospective studies. J Am Acad Dermatol 2001; 45: 520-7.

45. Odijk J, Kull I, Borres MP, et al. Breast-feeding and allergic disease: a multidisciplinary review of literature (1966-2001) on the mode of early feeding in infancy and its impact on later atopic manifestations. Allergy 2003; 58: 833-43.

46. Kull I, Bohme M, Borres MP, et al. Breast-feeding reduces the risk for childhood eczema. J All Clin Immunol 2005; 116: 657-61.

47. Saarinen UM, Kajosaari M. Breastfeeding as prophylaxis against atopic disease: prospective follow-up study until 17 years old. Lancet 1995; 346: 1065-9.

48. Oddy WH, Holt PG, Sly PD, et al. Association between breast feeding and asthma in 6 year old children: findings of a prospective birth cohort study. BJM 1999; 319: 815-9.

49. Harris JM, Cullinan P, Williams HC, et al. Environmental associations with eczema in early life. Br J Dermatol 2001; 144: 795-802.

50. Dunlop AL, Reichrtova E, Palcovicova L, et al. Environmental and dietary risk factors for infantile atopic eczema among a Slovak birth cohort. Pediatr Allergy Immunol 2006; 17: 103-11.

51. Ludvigsson JF, Mostrom M, Ludvigsson J, Duchen K. Exclusive breastfeeding and risk of atopic dermatitis in some 8300 infants. Pediatr Allergy Immunol 2005; 16: 201-8.

52. Bergmann RL, Diepgen TL, Kuss O, et al. Breastfeeding duration is a risk factor for atopic eczema. Clin Exp Allergy 2002; 32: 205-9.

53. Saarinen KM, Juntunen-Backman K, Jarvenpaa AL, et al. Breas-feeding and the development of cow's milk protein allergy. Adv Exp Med Biol 2000; 478: 121-30.

54. Vance GH, Lewis SA, Grimshaw KE, et al. Exposure of the faetus and infant to hen's egg ovalbumin via the placenta and breast milk in relation to maternal intake of dietary egg. Clin Exp Allergy 2005; 35: 1318-26.

55. Businco L, Marchetti F, Pellegrini G, et al. Prevention of atopic disease in "at-risk newborns" by prolonged breastfeeding. Ann Allergy 1983; 51: 296-9.

56. Chandra RK, Puri S, Suraiya C, Cheema PS. Influence of maternal food antigen avoidance during pregnancy and lacta- tion on incidence of atopic eczema in infants. Clin Allergy 1986; 16: 563-9.

57. Halken S, Host A, Jacobsen HP, et al. Prevention of food allergy in high-risk infants until the age of 5 years. A prospective dietary intervention study. Allergy 1995; 26: 49.

58. Hattevig G, Sigurs N, Kjellman B. Effects of maternal dietary avoidance during lactation on allergy in children at 10 years of age. Acta Paediatr 1999; 88: 7-12.

59. Sigurs N, Hattevig G, Kjellman B. Maternal avoidance of eggs, cow's milk and fish during lactation: effect on allergic manifestations, skin-prick-tests and specific IgE antibodies in children at age 4 years. Pediatrics 1992; 89: 735-9.

60. Høst A, Halken S, Muraro A, et al. Dietary prevention of allergic diseases in infants and small children. Pediatr Allergy Immunol 2008; 19: 1-4.

61. Halken S, Hansen KS, Jacobsen HP, et al. Comparison of a partially hydrolyzed infant formula with two extensively hydrolyzed formulas for allergy prevention: a prospective, randomized study. Pediatr Allergy Immunol 2000; 11: 149-61.

62. Halken S, Høst A, Hansen LG, Osterballe O. Preventive effect of feeding high-risk infants a casein hydrolysate formula or an ultrafiltrated whey hydrolysate formula. A prospective, randomized, comparative clinical study. Pediatr Allergy Immunol 1993; 4: 173-81.

63. Halken S, Høst A, Hansen LG, Osterballe O. Effect of an allergy prevention programme on incidence of atopic symptoms in infancy. A prospective study of 159 "high-risk" infants. Allergy 1992; 47: 545-53.

64. Schoetzau A, Filipiak-Pittro B, Franke K, et al. Effect of exclusive breast-feeding and early solid food avoidance on the incidence of atopic dermatitis in high-risk infants at 1 year of age. Pediatr Allergy Immunol 2002; 13: 234-42.

65. Remes ST, Castro-Rodriguez JA, Holberg CJ, et al. Dog exposure in infancy decreases the subsequent risk of frequent wheeze but not atopy. J Allergy Clin Immunol 2001; 108: 509-15.

66. Ownby DR, Johnson CC, Peterson EL. Exposure to dogs and cats in the first year of life and risk of allergic sensitization at 6 to 7 years of age. JAMA 2002; 288: 963-72.

67. Roost HP, Kunzli N, Schildler C, et al.; European Community Respiratory Health Survey. Role of current and childhood exposure to cat and atopic sensitization. J Allergy Clin Immunol 1999; 104: 941-7.

68. Mohrenschlager M, Darsow U, Schnopp C, et al. Atopic eczema: what's new? J Eur Acad Dermatol Venereol 2006; 20: 503-11.

69. Wen HJ, Chen PC, ChiangTL, et al. Predicting risk for early infantile atopic dermatitis by hereditary and environmental factors. BJD 2009; 161: 1166-72.

70. Cork MJ, Danby SG, Vasilopoulos Y, et al. Epidermal barrier dysfunction in atopic dermatitis. J Invest Dermatol 2009; 129: 1892-908.

71. Nowica D, Szepietowski J. Sucha skóra: przyczyny i pielęgnacja. Dermatol Estet 2006; 2: 97-9.

72. Wojowska D, Chodorowska G, Juszkiewicz-Borowiec M. Sucha skóra - patogeneza, klinika i leczenie. Postep Derm Alergol 2003; 20: 98-105.

73. Elias PM. Skin barrier function. Curr All Asth Rep 2008; 8: 299-305.

74. Niebuhr M, Mamerow D, Heratizadeh A, et al. Staphylococcal alpha-toxin induces a higher T cell proliferation and interleukin-31 in atopic dermatitis. Int Arch Allergy Immunol 2011; 156: 412-5. 
75. Lai Y, Cogen AL, Radek CA, et al. Activation of TLR2 by a small molecule produced by Staphylococcus epidermidis increases antimicrobial defense against bacterial skin infections. J Invest Dermatol 20510; 130: 2211-21.

76. Cho JS, Xuan C, Miller LS. Lucky number seven: RNasa 7 can prevent Staphylococcus aureus skin colonization. J Invest Dermatol 2010; 130: 2703-6.

77. Palmer DJ, Gold MS, Makrides M. Effect of cooked and raw egg consumption on ovalbumin content of human milk: a randomized, doubled-blind, cross-over trial. Clin Exp Allergy 2005; 35: 173-8.

78. Jarvinen KM, Makinen-Kiljunen S, Suomalainen H. Cow's milk challenge through human milk evokes immune responses in infants with cow's milk allergy. J Pediatr 1999; 135: 506-12.

79. Caubet JC, Eigenmann PA. Allergic triggers in atopic dermatitis. Immunol Allergy Clin N Am 2010; 30: 289-301.

80. Kaczmarski M, Wasilewska J, Jarocka-Cyrta E, et al. Polish statement on food allergy in children and adolescents. Postep Derm Alergol 2011; 28: 331-67.

81. Sigurs N, Hattevig G, Kjellmann B, Nilsson LBB. Appearance of atopic diseases in relation to serum IgE antibodies in children followed up from birth for 4 to 15 years. J Allergy Clin Immunol 1994: 94: 757-63.

82. Szepfalusi Z, Huber WD, Ebner C, et al. Early sensitization to airborn allergens. Int Arch Allergy Immunol 1995; 107: 595-8.

83. Kihlstrom A, Lilja G, Pershagen G, Hedlin G. Exposure to birch pollen in infancy and development of atopic diseases in childhood. J Allergy Clin 2002; 110: 78-84.

84. Breuer K, Wulf A, Constien A, et al. Birch pollen-related food as a provocation factor of allergic symptoms in children with atopic eczema/dermatitis syndrome. Allergy 2004; 59: 988-94.

85. Ortolani C, Pastorello EA, Farioli L, et al. IgE-mediated allergy from vegetable allergens. Ann Allergy 1993; 71: 470-6.

86. Reekers R, Schmidt P, Kapp A, Werfel T. Evidence of a lymphocyte response to birch pollen related food antigens in atopic dermatitis. J Allergy Clin Immunol 1999; 104: 466-72.

87. Yosipovitch G, Papoiu A. What causes itch in atopic dermatitis? Curr Allergy Asthma Rep 2008; 8: 306-11.

88. Arndt J, Smith N, Tausk F. Stress and atopic dermatitis. Curr Allergy Asthma Rep 2008; 8: 312-7. 